\title{
Comment concevoir la biosécurité des élevages porcins des milieux lacustres du sud Bénin pour réduire le risque de maintien et de dissémination de la peste porcine africaine?
}

\author{
Ogoudanan Ignace Dotché*, Karamatou Bio Seydou, Amen Gloria Gwladys Ahouanse, \\ Emmanuel Tohoun, Boris Constant Bankolé et Issaka Youssao Abdou Karim \\ Laboratoire de biotechnologie animale et de technologie des viandes, Département de production et santé animales, École polytechnique \\ d'Abomey-Calavi, Université d'Abomey-Calavi, 01 BP 2009, Cotonou, Bénin
}

\begin{abstract}
Résumé - L'élevage de porcs est pratiqué en milieu lacustre au Bénin malgré une forte présence d'eau de surface. L'étude vise à identifier les facteurs de risque de la dissémination de la peste porcine africaine (PPA) dans les pratiques d'élevage des milieux lacustres afin de proposer des mesures de biosécurité adaptées à ces milieux. Les données ont été collectées par entretien avec 48 éleveurs de porcs des communes des Aguégués et de Sô-Ava. Une typologie de ces élevages a permis d'identifier trois types d'élevage qui se distinguent sur le mode de logement, les types génétiques élevés, les types d'aliments utilisés, le mode de gestion des déchets et des cadavres des animaux. Le premier type correspond à des élevages en claustration totale qui ont des porcs de type génétique exotique nourris avec des mélanges de matières premières. Ces éleveurs jettent les déjections et les animaux morts dans l'eau du fleuve. Les deux autres sont des élevages en claustration partielle et en liberté qui ont des animaux de type génétique local nourris avec les restes de cuisine et du fourrage. Les déchets sont jetés dans l'espace non occupé par l'eau, mais des cadavres sont parfois enterrés (claustration partielle). Les pratiques d'élevage dans les trois types ne les protègent pas de la PPA. Cette vulnérabilité à la PPA des élevages diffère d'un type à l'autre et la liberté des porcs rend les élevages plus vulnérables. L'étude nous a permis de mettre en évidence une relation entre les différents types d'élevages et les risques de dissémination de la PPA, ce qui nous a conduits à élaborer une série de mesures de biosécurité adaptées à chaque type et destinées aux éleveurs et aux autorités.
\end{abstract}

Mots clés : porcin / PPA / pratique d'élevage / milieu lacustre

Abstract - How to conceive the biosecurity of pig farms in the lake areas of southern Benin to reduce the risk of maintenance and dissemination of African swine fever? Pig farming is practiced in lake environments of Benin despite a high presence of surface water. The study aims to identify risk factors for spreading of African swine fever (ASF) in lake environment breeding practices to propose biosecurity measures adapted to these environments. Data were collected by interview with 48 pig farmers in the communes of Aguégués and Sô-Ava. A typology of these farms has allowed us to identify three types of farms that differ in the type of housing, the genetic types reared, the feed types used, and the management of waste and dead animals. The first type corresponds to farms in total confinement that have pigs of exotic genetic type fed with mixtures of raw materials. These farmers throw the waste and dead animals into the river water. The other two types of farms are partially confined and free-range farms that have local genetic type animals fed with kitchen scraps and fodder. The waste is discharged into the space not occupied by water, but dead animals are sometimes buried (partial confinement). Breeding practices in all three types do not protect them from ASF. The vulnerability of the farms to ASF varies between types, and the free-ranging makes them more vulnerable to ASF. The study allowed us to identify a relationship between the different types of farms and the risks of ASF spreading, which led us to develop a series of biosecurity measures adapted to each type, both for farmers and authorities.

Keywords: pig / ASF / breeding practice / lake environment

\footnotetext{
*Auteur de correspondance : ogoudanan@yahoo.fr, dotcheign@gmail.com
} 


\section{Introduction}

Au Bénin, si les ruminants sont majoritairement élevés au nord du pays, la production porcine est plus développée au sud (Dotché et al., 2018). Dans cette zone géographique, deux types de biotopes sont rencontrés: le milieu que nous appellerons «terre ferme» dans lequel les habitats sont bâtis au sol et le milieu lacustre dans lequel l'eau occupe une grande partie et certains habitats sont construits sur l'eau (Djenontin, 2006a). Tout comme dans les communes à habitats sur sols, les porcs sont aussi élevés dans les milieux lacustres. Cette activité joue un rôle économique important de création de revenus pour les ménages. Contrairement aux élevages lacustres, les innovations technologiques issues des travaux de recherche et de développement sur l'élevage ont été diffusées sur terre ferme et ont contribué à améliorer les performances zootechniques des porcs à travers des croisements et l'amélioration des techniques d'élevage notamment l'alimentation, le suivi sanitaire, le logement et le choix des reproducteurs (Dotché et al., 2018, 2019; Kiki et al., 2019). Malheureusement, les progrès réalisés dans les élevages sont souvent neutralisés par la peste porcine africaine (PPA). En effet, les élevages, même quand ils intègrent les éléments d'amélioration, demeurent vulnérables. Entre les élevages extensifs et les élevages intégrant des améliorations techniques, on observe une diversité dans les modalités de conduite de l'élevage, ce qui amène à s'interroger sur la vulnérabilité de ces élevages face à la PPA.

Cette pathologie est apparue au Bénin pour la première fois en 1997 (Brown et al., 2018). Elle est causée par un virus à ADN de la famille des Asfaviridae qui se transmet par contact direct entre un animal sain et un animal malade puis par contact indirect par l'intermédiaire des fomites - les objets inanimés ou substances capables de transporter des organismes infectieux et de les transférer d'un individu à un autre, comme les déjections (Dixon et al., 2020). Entre 2003 et 2016, le pays a enregistré 236 épisodes de PPA avec plus de 20450 cas de mortalités (Couacy-Hymann, 2019). Les foyers de PPA sont plus signalés dans les milieux lacustres. C'est le cas de la commune des Aguégués où des foyers ont été signalés en 2017, 2018 et 2019 (DE, 2019).

L'absence de mesures de biosécurité dans les élevages pourrait justifier l'apparition de ces foyers. Cette absence de mesures de biosécurité semble liée à l'inadaptation des mesures classiques au milieu lacustre. Certaines pratiques observées dans les élevages sur terre ferme telles que l'absence de pédiluves dans les élevages, l'entrée des charcutiers et des visiteurs dans les élevages favorisent la circulation de la PPA dans ce milieu (Ohouko et al., 2020). De plus, les élevages lacustres peuvent constituer des réservoirs de recontamination des élevages sur terre ferme, car les éleveurs de ces deux milieux échangent des animaux vivants (Ohouko et al., 2020). L'objectif de cette investigation est en conséquence de décrire la diversité des formes d'élevage porcin en milieu lacustre afin d'identifier les pratiques qui peuvent favoriser le maintien et la dissémination de la PPA au sud du Bénin. Au terme de ce travail, seront proposées des mesures de biosécurité applicables dans les milieux lacustres tenant compte de cette diversité.

\section{Matériel et méthodes}

\subsection{Milieu d'étude}

Le Bénin est un pays de l'Afrique de l'Ouest. Il possède au sud un climat équatorial avec une alternance de deux saisons sèches (novembre à mars et mi-juillet à mi-septembre) et de deux saisons des pluies (avril à mi-juillet et mi-septembre à octobre). Au centre et au nord, le climat est de type tropical avec une saison sèche d'octobre à avril et une saison des pluies de mai à septembre.

L'étude a été réalisée dans les communes des Aguégués et de Sô-Ava au sud du pays. La commune des Aguégués est située au sud-ouest du département de l'Ouémé, sur une superficie de $103 \mathrm{~km}^{2}$. Cette commune lacustre est composée de plusieurs îlots d'accumulation alluviale logés dans la partie basse du fleuve Ouémé submergé par les crues de trois à cinq mois (juillet à novembre) par an. Elle présente un climat de type équatorial humide caractérisé par deux saisons des pluies et deux saisons sèches d'importance inégale. La pluviométrie moyenne annuelle est de $1200 \mathrm{~mm}$. Le principal cours d'eau qui traverse les Aguégués est le delta de l'Ouémé (Djenontin, 2006b).

La commune de Sô-Ava, commune lacustre du sud Bénin, occupe la basse vallée du fleuve Ouémé et de la rivière Sô. Une partie de cette commune se trouve aussi sur le lac Nokoué. D'une superficie de $209 \mathrm{~km}^{2}$, elle jouit du même climat que la commune des Aguégués. Les sols sont composés de sable d'origine marine avec en profondeur de l'argile vaseuse et des alluvions provenant de la vallée de l'Ouémé (Djenontin, 2006a).

\subsection{Méthodologie}

La méthodologie utilisée est celle d'une enquête rétrospective par entretien avec les éleveurs. L'absence d'une liste des éleveurs de la localité nous a obligés à nous rapprocher de l'agence territoriale du développement agricole qui nous a mis en contact avec les premiers éleveurs. Ensuite, nous avons utilisé la méthode de la "boule de neige» qui s'arrête lorsque nous retombons sur les éleveurs déjà interrogés pour retrouver les autres. Nous avons interrogé tous les éleveurs (48 personnes au total) dont nous avons eu connaissance. Toutefois, certains éleveurs non cités peuvent ne pas avoir été pris en compte. Une fiche d'enquête a été utilisée pour collecter les données. Les éleveurs ont été interrogés sur le mode d'élevage (en liberté et claustration), l'ethnie, les activités, le niveau d'étude, le titre de propriété, les objectifs de production, les difficultés rencontrées, les génotypes des porcs élevés, la structure du cheptel, le mode de constitution du cheptel, les paramètres techniques, le mode de logement, l'alimentation, le suivi sanitaire, les pathologies rencontrées, la gestion des déjections et des cadavres. Les animaux de génotype exotique regroupent les probables races exotiques pures et les produits de divers croisements réalisés entre ces races.

\subsection{Analyses statistiques}

La fonction MCA de la librairie FactoMineR de $R$ a été utilisée pour l'analyse des correspondances multiples (ACM). 


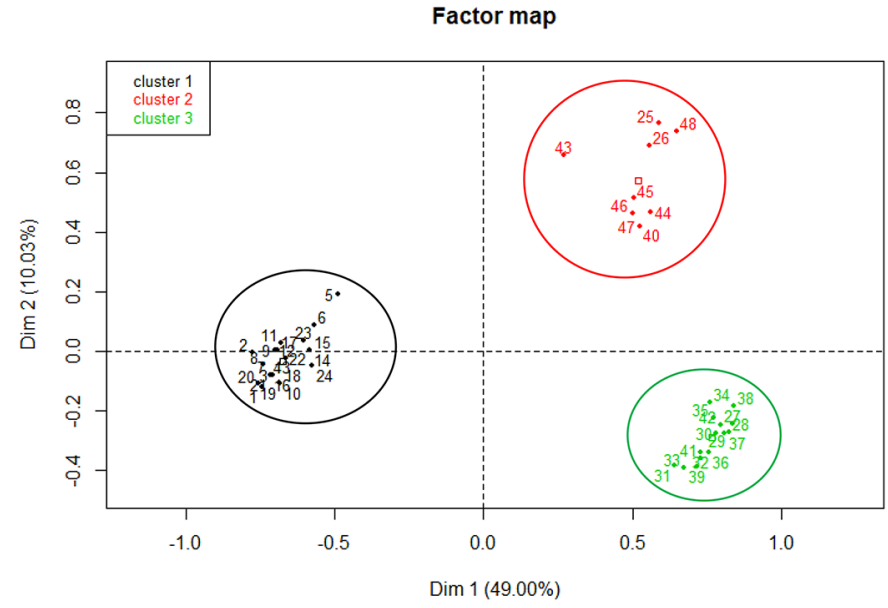

Fig. 1. Typologie des élevages de porcs des milieux lacustres du Bénin.

Fig. 1. Typology of pig farms in lake environments in Benin.

Les variables prises en compte dans l'analyse des correspondances sont les activités des éleveurs, les génotypes élevés, le mode de logement des porcs, les types d'aliments, les pathologies rencontrées, la période d'apparition des pathologies, le mode de traitement des maladies et le mode de gestion des cadavres et des déjections. Une classification ascendante hiérarchique sur les composantes de l'ACM a été ensuite réalisée par la fonction HCPC. Des groupes d'éleveurs ont été identifiés et chaque groupe correspond à un type d'élevage. Ces types ont été ensuite caractérisés en testant l'effet du type d'élevage par le test de $F$ sur les variables quantitatives et par le test de $\mathrm{Chi}^{2}$ sur les variables qualitatives. Les fréquences ont été comparées deux à deux entre les types par le test bilatéral de $Z$. Pour chaque fréquence relative, un intervalle de confiance à $95 \%$ a été calculé suivant la formule :

$$
I C=1,96 \sqrt{\frac{[P(1-P)]}{N}},
$$

où $P$ est la fréquence relative et $N$ la taille de l'échantillon.

\section{Résultats}

\subsection{Typologie des élevages de porcs dans les milieux lacustres}

Trois axes ont été retenus pour l'interprétation des résultats de l'ACM. Ces trois axes représentent $64 \%$ de la variabilité des données, dont $49 \%$ sur le premier axe, $10 \%$ sur le deuxième axe et $5 \%$ sur le troisième axe. Chaque axe a permis de décrire un profil d'élevage ou type d'élevage. La figure 1 présente les trois groupes d'élevages formés dans l'espace à 3 dimensions et visualisés dans le plan formé par les deux premières dimensions. Les élevages de type 1 ont été décrits uniquement dans les Aguégués et ceux des types 2 et 3 à Sô-Ava. Le groupe 1 est composé de 24 personnes et les groupes 2 et 3 respectivement de 9 et 15 .

Dans l'élevage du type 1 dénommé élevage en claustration totale, les éleveurs interrogés élèvent des porcs de génotype exotique dans des logements en bois construits sur pilotis. Les animaux sont gardés en claustration totale toute l'année. Ils sont nourris avec des mélanges de quelques matières premières achetées au marché et auprès des transformatrices des produits agricoles. Les éleveurs de ce groupe jettent les animaux morts dans le fleuve.

Dans le type 2 dénommé élevage en claustration partielle, les éleveurs ont des porcs de génotype local. Ils disposent des logements en bois et en dur construits au sol dans les zones non occupées par l'eau. Les personnes interrogées pratiquent l'élevage en claustration partielle dans lequel les porcs sont enfermés pendant la saison des pluies. Les porcs sont nourris avec les restes de cuisine et des fourrages. En cas de mortalité d'un animal, l'éleveur jette son cadavre dans l'environnement, l'enterre ou le consomme.

Dans le type 3 désigné par élevage en liberté, les éleveurs ne disposent pas de logements et les porcs (génotype local) sont en liberté toute l'année dans la commune. Les porcs de ce type d'élevage se nourrissent de résidus et de restes d'aliments trouvés sur les tas d'ordures et de fourrages. Les animaux morts dans ce type sont abandonnés sur les tas d'ordures.

\subsection{Caractéristiques des types d'élevage de porcs en milieu lacustre au Bénin}

\subsubsection{Statut et objectifs de production des éleveurs}

Les personnes pratiquant l'élevage en claustration totale sont en majorité des hommes ( $83 \%$ ) et d'ethnie Goun. En revanche, la majorité des éleveurs pratiquant la claustration partielle $(67 \%)$ et la liberté $(80 \%)$ sont des femmes et d'ethnie Ayizo. Ils sont tous de religion chrétienne et en majorité non scolarisés $(71 \%)$. La majorité des éleveurs du mode claustration totale pratique la pêche $(41 \%)$ comme principale activité génératrice de revenus. Dans les modes en claustration partielle et en liberté, la majorité des femmes $(56 \%$ et $80 \%)$ retrouvées dans cette activité sont des ménagères. Les autres activités pratiquées par les personnes interrogées sont l'agriculture, l'élevage, les emplois de fonctionnaires, le commerce, l'artisanat et les travaux occasionnels. Les porcs appartiennent au chef du ménage $(79 \%)$ ou à sa femme $(21 \%)$ dans les élevages en claustration totale alors qu'ils appartiennent à son épouse $(100 \%)$ dans les deux autres types.

L'objectif des éleveurs est de produire des porcs pour la vente même si une partie peut être auto-consommée par la famille. Les catégories d'âge souvent vendues par les éleveurs sont des porcelets destinés à la reproduction (dès après le sevrage) et les porcs engraissés. En dehors de ces catégories, certains éleveurs vendent des verrats et des truies. $\mathrm{La}$ proportion des éleveurs qui vendent des truies dans les modes en claustration partielle $(33 \%)$ et en liberté $(40 \%)$ est supérieure $(p<0,01)$ à celle des élevages en claustration totale $(4 \%)$. Les verrats en reproduction sont vendus seulement dans les élevages en claustration partielle et en liberté.

\subsubsection{Génotypes élevés et structure du cheptel}

Le génotype des porcs élevés par les éleveurs varie $(p<0,001)$ d'un type à un autre. Ainsi, tous les éleveurs qui gardent les porcs en claustration partielle et en liberté déclarent qu'ils détiennent des porcs de génotype local et des croisés, alors que la majorité de ceux du mode claustration totale ( $96 \%$ ) élève un génotype exotique et $4 \%$ le génotype local et des croisés. 


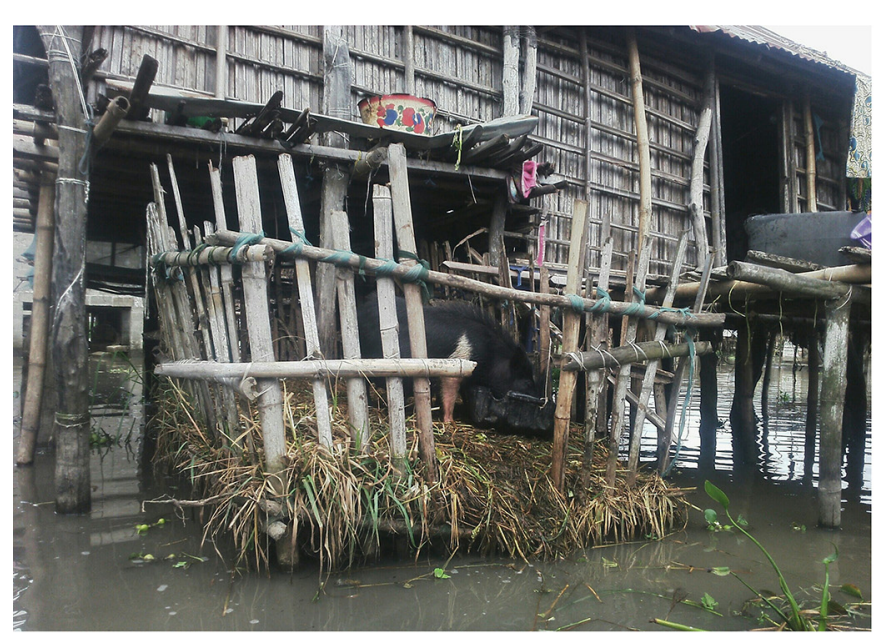

(a)

Fig. 2. Logements des porcs en milieu lacustre.

Fig. 2. Housing of pigs in the lake area.

L'effectif moyen de porcs par cheptel est de 5,5 têtes dans les élevages en claustration totale, 5 dans les élevages en claustration partielle et 8 dans les élevages en liberté. Ce cheptel est composé de 0,3 verrat, 1 femelle et 4,2 jeunes (avant l'âge d'entrée en reproduction) dans les élevages en claustration totale. Dans les élevages en claustration partielle, ce cheptel est constitué de 0,2 verrat, 0,9 truie et 4 jeunes. Le cheptel dans les élevages en liberté est composé de 1,9 truie et de 6,1 jeunes. Le nombre de truies enregistré dans les élevages en liberté est supérieur $(p<0,05)$ à celui des élevages en claustration (totale et partielle).

\subsubsection{Modes de conduite de l'élevage en milieu lacustre \\ 3.2.3.1 Logement des porcs}

Les porcs sont élevés dans un système qualifié de «traditionnel» par les éleveurs eux-mêmes dans les deux communes. Le mode de logement des porcs varie $(p<0,001)$ d'un type d'élevage à l'autre. Tous les éleveurs pratiquant la claustration (100\%) disposent de logements pour les animaux (Fig. 2). En revanche, les élevages en liberté n'ont pas de logements pour les porcs (Tab. 1). Les logements sont construits sur pilotis dans le mode en claustration totale, alors qu'ils sont érigés directement au sol dans le mode en claustration partielle. Le bois est le matériau le plus utilisé dans la construction des logements (Tab. 1). Certains logements disposent de toits en tôle ou en paille et d'autres sont sans toit. Les toits en tôle sont plus rencontrés $(p<0,05)$ dans les élevages en claustration totale que dans les élevages en claustration partielle. La proportion de logements sans toits dans les élevages en claustration partielle (33\%) est supérieure $(p<0,05)$ à celle des élevages en claustration totale $(4 \%)$. Les animaux en claustration partielle (Fig. 3) sont gardés enfermés en permanence pendant la saison des pluies et sont en liberté en saison sèche.

\subsubsection{Alimentation des porcs}

Les éleveurs utilisent des mélanges de sous-produits agricoles, des restes de cuisine et des fourrages pour nourrir les

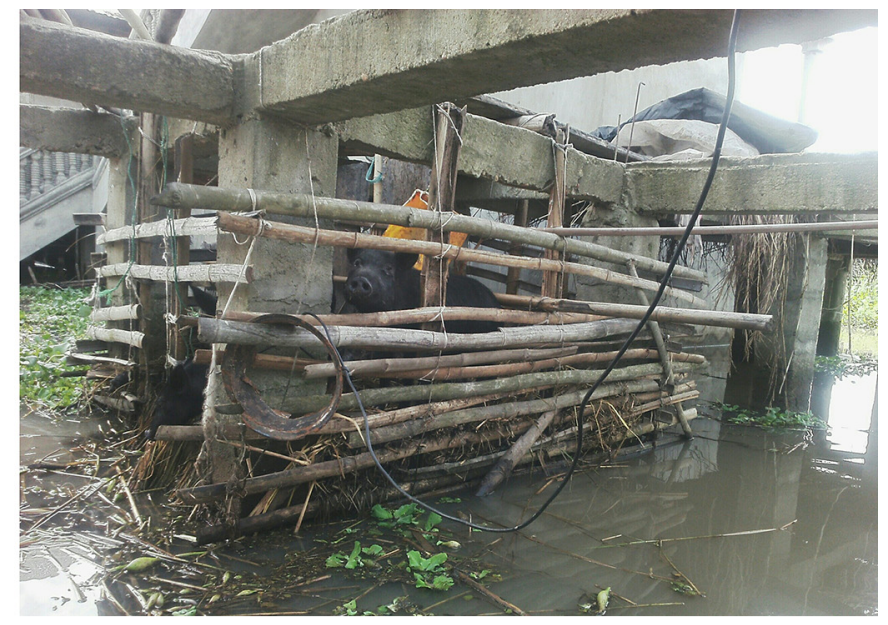

(b)

porcs (Tab. 2). Les éleveurs qui gardent les porcs en claustration totale utilisent plus souvent $(p<0,001)$ les mélanges de sous-produits agricoles que ceux du mode en claustration partielle. En revanche, les fourrages se donnent davantage $(p<0,001)$ aux porcs en claustration partielle et en liberté qu'à ceux en claustration totale. Dans les trois types, les sous-produits utilisés sont principalement les sons de céréales (maïs, riz) et le son de soja (Tab. 2). Les éleveurs pratiquant la claustration totale achètent les sous-produits alors que ceux pratiquant la claustration partielle (44\%) et la liberté (60\%) produisent leurs propres sous-produits par transformation artisanale de maïs en akassa et de soja en fromage. Dans les deux communes, l'eau des forages est utilisée pour abreuver les porcs. Toutefois, les animaux en liberté peuvent aussi s'abreuver au fleuve.

\subsubsection{Suivi sanitaire et difficultés rencontrées}

Les éleveurs ne disposent pas de plans de prophylaxie. Le suivi sanitaire consiste seulement en un nettoyage des loges des porcs en claustration. La gestion des déjections évacuées diffère d'un type à l'autre. Dans les élevages en claustration totale, ces déjections sont déversées dans le fleuve alors que les éleveurs en claustration partielle les jettent sur les espaces non occupés par l'eau.

Les pathologies sont les difficultés majeures auxquelles sont confrontés tous les éleveurs (Tab. 3). En dehors des pathologies, les éleveurs en claustration totale ont signalé des difficultés alimentaires. La pathologie la plus enregistrée par les éleveurs est la PPA. Toutes les personnes interrogées ont déclaré qu'elles ont connu au moins une fois la PPA dans leur élevage. Ces éleveurs ont également signalé la présence d'ectoparasites, surtout la gale sarcoptique, dans leurs élevages. Ces pathologies apparaissent en saison pluvieuse dans la plupart des élevages. Les porcs malades sont soignés avec des produits vétérinaires dans les élevages en claustration totale $(92 \%)$ et avec la pharmacopée traditionnelle dans les élevages en claustration partielle et en liberté. En cas d'échec des traitements, les éleveurs pratiquant la claustration totale $(45 \%)$ vendent les animaux. 
Tableau 1. Conditions de logement des porcs en milieu lacustre.

Table 1. Habitat and housing of pigs in the lake area.

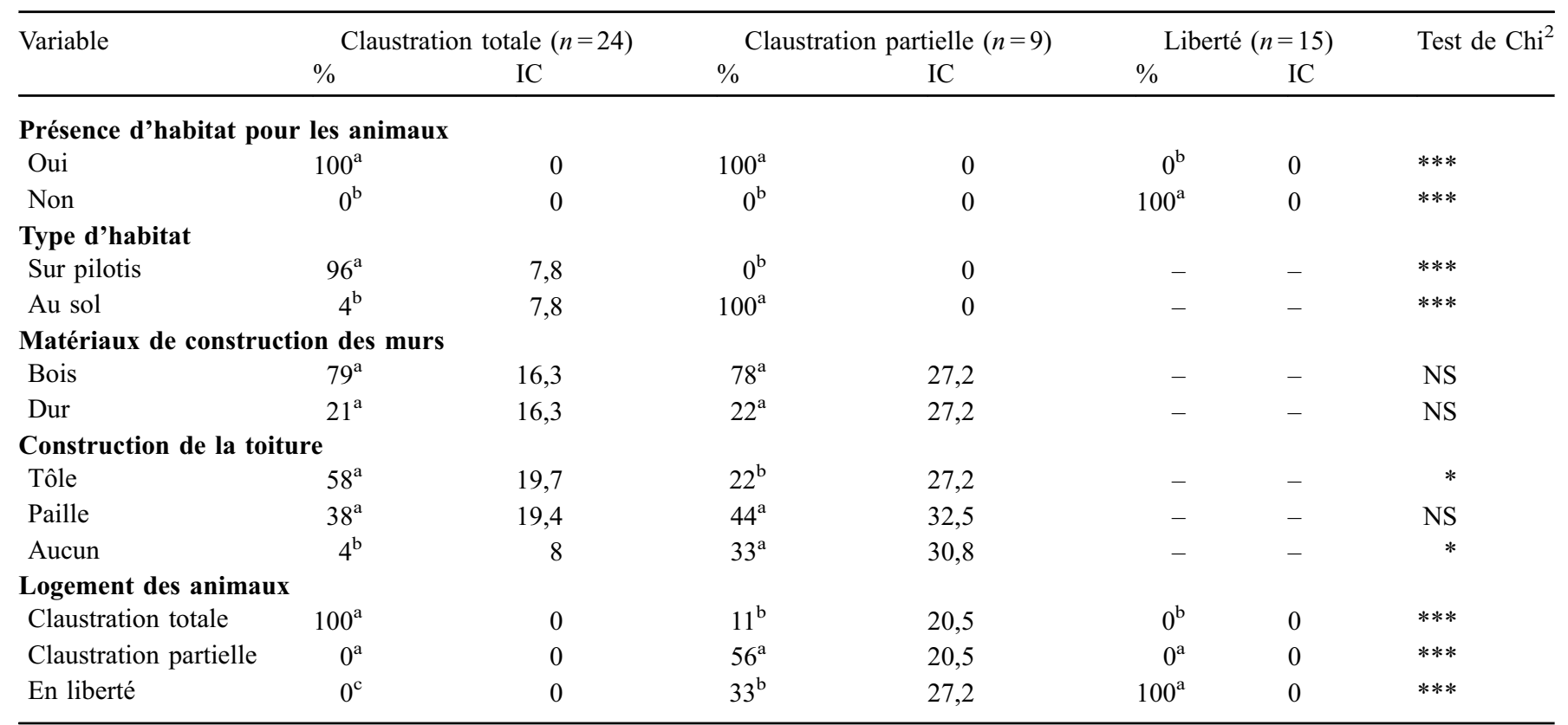

IC : intervalle de confiance; NS : $p>0,05 ; *: p<0,05 ; * * *: p<0,001 ;^{\text {a,b }}:$ les pourcentages sur une même ligne suivis de lettres différentes diffèrent significativement au seuil de $5 \%$.

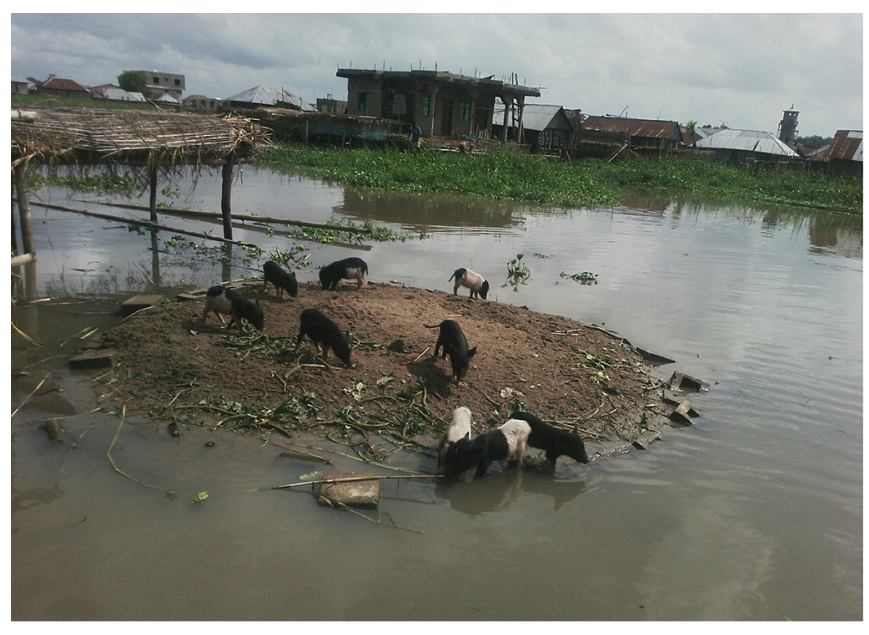

Fig. 3. Porcs en divagation en milieu lacustre au Bénin.

Fig. 3. Pigs in free-range in a lake environment in Benin.

La PPA est la principale cause de mortalité des porcs selon les éleveurs. Tous les éleveurs pratiquant la claustration partielle et la liberté et $89 \%$ de ceux en claustration totale ont affirmé que tous leurs animaux meurent en cas d'épisode de PPA. En cas de mortalité, les élevages en claustration totale jettent les cadavres des animaux dans l'eau du fleuve. Dans les élevages en claustration partielle et en liberté, les cadavres des animaux sont abandonnés sur les tas d'ordures, consommés, jetés dans l'eau du fleuve, ou encore enterrés (22\%) (Tab. 3). La proportion de ceux qui jettent les cadavres dans l'eau du fleuve dans les élevages en claustration totale est supérieure $(p<0,001)$ à celle des élevages en liberté.

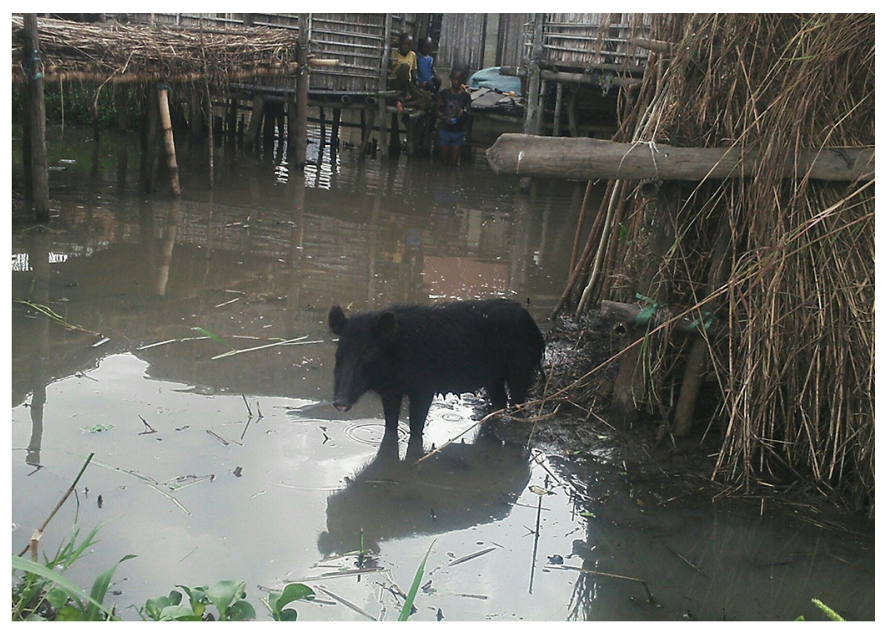

\subsection{Performances zootechniques des animaux élevés}

La taille des portées des porcs de génotype exotique des élevages en claustration totale $(9,1$ porcelets) est plus élevée $(p<0,001)$ que celle du génotype local des élevages en claustration partielle $(7,2)$ et en liberté $(5,1)$. La taille des portées du génotype local des élevages en claustration partielle est aussi plus élevée $(p<0,001)$ que celle des élevages en liberté. Le taux de mortinatalité est de $0 \%$ dans les élevages en claustration totale, $0,8 \%$ dans les élevages en claustration partielle et 2,6\% dans les élevages en liberté. Le taux de mortalité entre la naissance et le sevrage est de 5,8\% dans les 
Tableau 2. Alimentation des porcs.

Table 2. Pig feeding.

\begin{tabular}{|c|c|c|c|c|c|c|c|}
\hline \multirow[t]{2}{*}{ Variable } & \multicolumn{2}{|c|}{ Claustration totale $(n=24)$} & \multicolumn{2}{|c|}{ Claustration partielle $(n=9)$} & \multicolumn{2}{|c|}{ Liberté $(n=15)$} & \multirow[t]{2}{*}{ Test de $\mathrm{Chi}^{2}$} \\
\hline & $\%$ & $\mathrm{IC}$ & $\%$ & $\mathrm{IC}$ & $\%$ & $\mathrm{IC}$ & \\
\hline \multicolumn{8}{|l|}{ Type d'aliments } \\
\hline Résidus et reste de cuisine & $100^{\mathrm{a}}$ & 0 & $100^{\mathrm{a}}$ & 0 & $100^{\mathrm{a}}$ & 0 & NS \\
\hline Fourrages & $4^{\mathrm{b}}$ & 8 & $44^{\mathrm{a}}$ & 32,5 & $60^{\mathrm{a}}$ & 24,8 & $* * *$ \\
\hline Sons de céréales & $100^{\mathrm{a}}$ & 0 & $100^{\mathrm{a}}$ & 0 & $100^{\mathrm{a}}$ & 0 & NS \\
\hline \multicolumn{8}{|l|}{ Provenance des matières premières } \\
\hline Achat & $100^{\mathrm{a}}$ & 0 & $44^{\mathrm{b}}$ & 32,5 & $60^{\mathrm{b}}$ & 24,8 & $* * *$ \\
\hline Production & $0^{\mathrm{b}}$ & 0 & $56^{\mathrm{a}}$ & 32,5 & $40^{\mathrm{a}}$ & 24,8 & $* * *$ \\
\hline
\end{tabular}

IC : intervalle de confiance; NS : $p>0,05 ; * * *: p<0,001 ;{ }^{\mathrm{a}, \mathrm{b}}:$ les pourcentages sur une même ligne suivis de lettres différentes, diffèrent significativement au seuil de $5 \%$.

Tableau 3. Difficultés rencontrées et suivi sanitaire.

Table 3. Difficulties encountered and health monitoring.

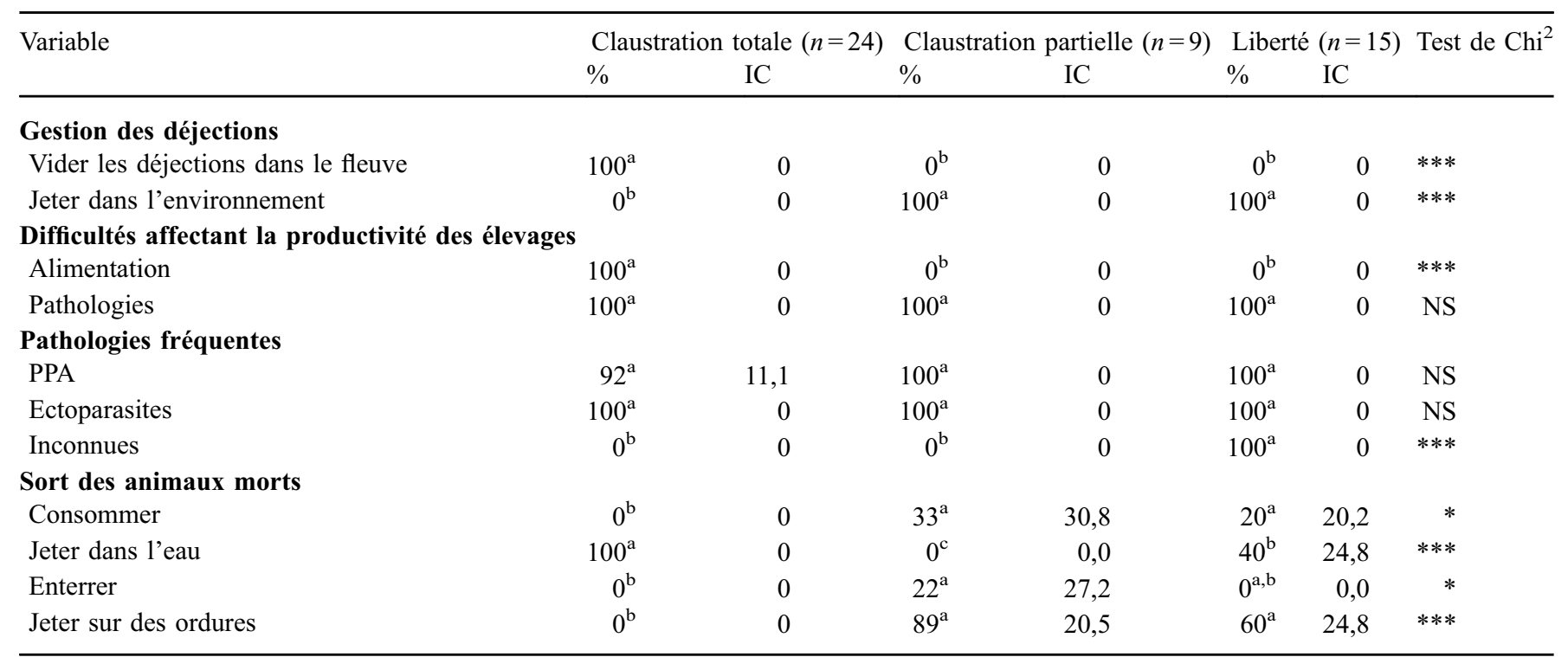

IC : intervalle de confiance; NS : $p>0,05 ; *: p<0,05 ; * * *: p<0,001 ;{ }^{\mathrm{a}, \mathrm{b}}$ : les pourcentages sur une même ligne suivis de lettres différentes, diffèrent significativement au seuil de $5 \%$.

élevages en claustration totale, $6,1 \%$ dans les élevages en claustration partielle et $6,7 \%$ dans les élevages en liberté. Le nombre moyen de porcelets sevrés par truie de génotype exotique dans les élevages en claustration totale $(8,5$ porcelets) est supérieur $(p<0,001)$ à celui des truies de génotype local des élevages en claustration partielle ( 6,7 porcelets) et en liberté $(4,8$ porcelets). Le nombre de porcelets sevrés dans les élevages en claustration partielle est plus grand que celui des élevages en liberté. Les porcelets de génotype local sont sevrés à un âge plus avancé $(p<0,01)$ dans les élevages en claustration partielle (86 jours) que les porcelets de génotype exotique des élevages en claustration totale (65 jours).

\section{Discussion}

\subsection{Diversité des systèmes d'élevage liée à la diversité des acteurs}

\subsubsection{Statut des éleveurs et génotypes élevés}

L'observation d'élevages uniquement en claustration complète dans les Aguégués est contraire au constat de Tohoun (2017), qui avait rapporté l'existence d'élevages en claustration partielle dans cette zone. L'élimination des élevages en claustration partielle dans les Aguégués est liée aux épisodes de PPA entre 2017 et 2019, qui ont entraîné la mort de plus de 10000 porcs (DE, 2019), ce qui a découragé les 
éleveurs de ce type. En dehors de la mortalité des animaux, la peur de perdre les porcs, qui oblige les éleveurs à les vendre en cas d'annonce de cas de PPA dans les élevages voisins, pourrait aussi être à l'origine de cette disparition. Toutefois, la disparition des élevages en claustration partielle dans les Aguégués pourrait n'être que temporaire et ces élevages pourraient réapparaître après l'absence prolongée d'annonce de cas de PPA. La persistance des élevages en claustration totale ne démontre pas une capacité de résistance de ces élevages à la PPA, mais traduit la reprise rapide de l'élevage après chaque épisode de PPA. Ces éleveurs sont obligés de reprendre l'activité à cause des investissements élevés liés à ce type (logements) et de la nécessité de les amortir. Des mortalités ont été aussi signalées à Sô-Ava, mais le nombre de cas semble être plus faible que dans les Aguégués (DE, 2019). Il est toutefois possible que, dans les Aguégués, des élevages en liberté aient échappé à notre étude, mais ces élevages doivent être très peu présents pour ne pas être remarqués. L'absence de l'élevage en claustration totale à Sô-Ava pourrait être liée à un manque de soutien des organisations de développement de l'élevage pour l'amélioration des systèmes dans cette commune. L'implication majoritaire des femmes dans l'élevage de porcs à Sô-Ava est liée à l'élevage en liberté pratiqué par la majorité des personnes interrogées $(62 \%)$ de cette commune. Ce mode d'élevage ne nécessite pas un grand investissement, comparativement à l'élevage en claustration totale qui nécessite des moyens financiers conséquents pour la construction des logements et l'achat des sous-produits pour l'alimentation des animaux, ainsi que pour l'achat d'animaux de race exotique.

Dans les Aguégués, l'implication minoritaire des femmes dans l'élevage des porcs serait liée à une perte de motivation engendrée par les mortalités associées à la PPA pour cet élevage, car Tohoun (2017) avait rapporté $48 \%$ de femmes impliquées dans l'élevage porcin dans cette commune contre $17 \%$ seulement dans notre étude. Cette implication minoritaire peut aussi s'expliquer par le manque de moyens financiers des femmes pour investir dans les modèles de logements utilisés par les hommes dans cette commune.

Les éleveurs des modes en claustration partielle et en liberté détiennent davantage de porcs de génotype local car ils pratiquent davantage l'élevage en liberté. Ce mode de conduite des porcs convient mieux à ce génotype local, plus rustique, qu'aux porcs de génotype exotique qui supportent difficilement les aliments médiocres et les pathologies rencontrées dans ce système (Kiki et al., 2018). La présence majoritaire de porcs de génotype local dans les élevages en liberté a déjà été rapportée au Bénin et au Sénégal (Dotché et al., 2018; Ossebi et al., 2018) et celle d'animaux de génotype exotique dans les élevages «améliorés» au Bénin (Dotché et al., 2018). Toutefois, certaines personnes gardent les porcs de génotype local même s'ils choisissent d'élever en claustration totale. Les performances zootechniques du génotype local sont cependant meilleures dans les élevages en claustration que dans le système extensif (Kouthinhouin et al., 2009), mais malheureusement, selon les éleveurs de notre étude, le gain réalisé suffit rarement à couvrir les dépenses liées à l'alimentation en claustration. Ce constat des éleveurs est lié au fait que les travaux d'amélioration de l'élevage du génotype local visent à pouvoir l'élever dans les conditions d'élevage du génotype exotique, sans tenir compte de ses potentialités propres (valorisation de ressources alimentaires médiocres, résistance aux pathologies).

\subsubsection{Mode de conduite de l'élevage}

L'élevage en liberté est lié au profil des éleveurs de ce groupe, qui sont des femmes à faibles ressources financières. Ce système est amélioré dans les élevages en claustration par la présence de logement. Toutefois, les éleveurs de ces types (claustration partielle et totale) qualifient ce système de « traditionnel » à cause des matériaux précaires qu'ils utilisent pour la construction des logements (surtout dans la claustration partielle) tels que le bois, les claies, les feuilles de palmiers, les tôles réutilisées, les pailles. L'analyse des deux pratiques d'élevage en claustration montre que l'élevage en claustration totale est comparable à l'élevage amélioré décrit par Kiki et al. (2018) dans l'Ouémé, et l'élevage en claustration partielle à l'élevage traditionnel décrit par Agbokounou et al. (2016).

L'absence de plan de prophylaxie dans les élevages explique la forte présence des pathologies signalée par les éleveurs. Cette situation pourrait s'aggraver avec le déversement des déjections et des cadavres dans l'eau de la rivière. Les pathologies enregistrées sont la PPA, les ectoparasites et d'autres pathologies dont ils ignorent le nom. Ces pathologies ont été déjà rapportées dans les élevages de porcs dans divers travaux menés au sud Bénin (Kiki et al., 2018). Les pathologies apparaissent en saison des pluies parce qu'à cette période l'eau inonde le milieu et l'on assiste alors à une pollution microbienne. Les femmes des élevages menés en claustration partielle et en liberté utilisent la pharmacopée traditionnelle pour traiter les porcs parce qu'elles ne disposent pas de moyens financiers suffisants pour l'achat des produits vétérinaires utilisés par les hommes ayant des élevages en claustration totale.

Sur la base de nos résultats et de ceux de Tohoun (2017), nous constatons que l'effet d'élimination lié à la PPA a réduit la diversité des élevages dans les Aguégués, ce qui a pour conséquence la disparition du génotype local et la réduction des activités des femmes. L'amélioration de l'élevage en liberté à Sô-Ava est donc indispensable pour éviter que se produise leur élimination comme dans les Aguégués. Pour améliorer la conduite de l'élevage et préserver à la fois les activités des femmes et la diversité génétique, il nous semble pertinent de réduire la liberté des porcs, sans l'éliminer complètement.

\subsection{Implication de la diversité des systèmes d'élevage de porc en milieu lacustre dans le maintien et la dissémination de la PPA}

Le milieu lacustre combiné à certaines pratiques d'élevage maintient et dissémine les pathologies dans les élevages porcins de cet espace lacustre, et pourrait être responsable de leur diffusion dans les élevages situés sur la terre ferme.

Le maintien de la PPA dans le milieu lacustre est lié aux pratiques d'élevage et à la présence d'eau. Ainsi, l'élevage en liberté peut contribuer au maintien de la PPA dans le milieu, car les contacts entre les porcs malades et les porcs sains sont fréquents avec ce mode de conduite. Ces contacts peuvent survenir lors de l'alimentation, mais aussi en cas 
d'accouplement, car certains éleveurs n'ont pas de verrat et les truies de ces élevages sont saillies en liberté. Ce risque de contamination est plus élevé s'il existe des porteurs sains dans le milieu (Eblé et al., 2019). L'implication des porcs en liberté dans la diffusion de la PPA a été déjà notée dans les élevages de porcs en Afrique (Penrith et al., 2013 ; Brown et al., 2018 ; Fasina et al., 2020). Les animaux en liberté pourraient aussi être contaminés par la faune sauvage (surtout les phacochères) (Penrith et al., 2013). Toutefois, ce dernier mode de transmission n'est pas plausible ici, car les phacochères ne se rencontrent pas dans ce milieu lacustre au Bénin.

Dans les trois types d'élevage, les pratiques alimentaires peuvent faciliter la circulation du virus. Ainsi, les sousproduits agricoles (élevage en claustration), les restes de récoltes et de cuisine (tous les types) et les fourrages (élevages en claustration partielle et en liberté) ne sont pas traités thermiquement avant d'être utilisés et cela constitue un risque d'introduction du virus dans l'élevage, car ces ressources sont manipulées par diverses personnes (lors de la production, au marché, lors du transport, etc.) avec la possibilité qu'elles soient contaminées par au moins une personne. La contamination des porcs à travers les aliments a été déjà rapportée en Afrique (Penrith et al., 2013). C'est pourquoi, Dixon et al. (2020) suggèrent aux éleveurs de traiter thermiquement ces ressources alimentaires afin d'inactiver le virus avant leur utilisation.

La pratique qui consiste à jeter les cadavres et surtout ceux des animaux morts de la PPA (tous les types d'élevage), et les déchets issus du traitement de ces cadavres (élevages en claustration partielle et en liberté) dans l'environnement ne peuvent que favoriser la diffusion de l'agent pathogène, car les animaux en liberté cherchent leur nourriture dans les mêmes endroits que ceux où ces cadavres sont jetés. Cette possibilité de contamination des porcs à travers l'environnement a été déjà démontrée par Mazur-Panasiuk et al. (2019) car, selon ces auteurs, le virus de la PPA persiste dans les cadavres et les déchets jetés dans l'environnement. La pratique qui consiste à enterrer les cadavres, utilisée dans les élevages en claustration partielle, est une bonne pratique, mais elle doit être améliorée par l'usage de la chaux vive lors de l'enfouissement des cadavres de façon à assurer leur élimination complète. De plus, les éleveurs devraient veiller à enterrer les animaux morts dans les endroits non inondables afin que l'eau ne provoque pas leur désenfouissement.

La pirogue utilisée pour accéder aux porcheries dans les élevages en claustration (partielle et totale) en période de crue peut être un vecteur de contamination des élevages, car la même pirogue est utilisée pour les déplacements de la famille, des charcutiers et des porcs. La contamination indirecte des porcs à travers le matériel de transport a été signalée par Penrith et al. (2013). Les vêtements, chaussures et les matériels des éleveurs peuvent aussi être des contaminants indirects des porcs (Penrith et al., 2013; Fasina et al., 2020).

En dehors de ces facteurs liés aux pratiques d'élevage, la présence de l'eau dans le milieu peut jouer un rôle dans la dissémination du virus de la PPA. L'eau peut en effet être contaminée par les cadavres qui y sont jetés directement (élevage en claustration totale), mais aussi par ceux jetés dans l'environnement et probablement par ceux mal enterrés. Cette eau contaminée infecte les porcs en claustration en cas d'inondation et les porcs en liberté lorsqu'ils s'abreuvent ou s'alimentent. Il n'y a pas de travaux scientifiques prouvant que le virus de la PPA subsiste dans l'eau de la zone lacustre étudiée, mais des travaux réalisés en Europe et en Russie montrent que la présence d'eau de surface est associée à la présence de la PPA (Gulenkin et al., 2011; Martínez-López et al., 2015), ce qui peut faire penser à un probable rôle de l'eau dans l'apparition et la transmission de la PPA. Pour éclaircir le rôle de l'eau en tant que vecteur, il sera nécessaire de réaliser des études pour rechercher la présence du virus dans l'eau du fleuve.

Ainsi, les élevages lacustres pourraient servir de réservoirs pour les élevages situés sur la terre ferme. La contamination des élevages de la terre ferme pourrait se faire soit par l'eau, soit par les transactions entre les deux formes d'élevage (lacustre et terre ferme). L'eau du fleuve s'écoule ensuite vers d'autres communes où surviennent aussi des inondations, ce qui fait que le virus peut être transporté par l'eau des élevages lacustres vers ces localités. Les porcs produits en milieu lacustre sont enfin souvent vendus aux charcutiers des zones sur terre ferme et ces derniers peuvent contaminer les élevages de leurs zones après manipulation d'un animal malade de la PPA, comme rapporté par Costard et al. (2009). En sens inverse, les éleveurs des zones lacustres achètent des animaux de renouvellement dans les élevages sur terre ferme. Les éleveurs peuvent donc s'échanger le virus pendant ces transactions, comme l'ont mentionné Fasina et al. (2020) en Tanzanie.

\subsection{Quelques recommandations de biosécurité pour réduire la vulnérabilité à la PPA en milieu lacustre}

Les points essentiels des mesures habituellement recommandées pour empêcher la circulation de la PPA dans les élevages concernent: la mise en quarantaine de nouveaux porcs achetés par l'éleveur, le changement des vêtements avant d'entrer dans l'exploitation, l'utilisation de pédiluves pour désinfecter les bottes, la désinfection des camions de transport, le confinement des animaux et la non-utilisation des eaux grasses et des aliments les contenant (Penrith et al., 2013; Dixon et al., 2020). La présence d'eau en milieu lacustre fait que ces mesures nécessitent des adaptations afin d'être applicables dans les élevages concernés.

Les mesures à mettre en œuvre exigent des actions de la part des éleveurs, mais aussi des autorités locales. Les éleveurs des trois types devraient ainsi traiter thermiquement les ressources alimentaires utilisées et éviter de jeter les cadavres dans l'eau du fleuve et dans l'environnement. Les pédiluves utilisés dans les élevages sur terre ferme (Asambe et al., 2019) ne peuvent pas être utilisés en milieu lacustre; il faudrait que les éleveurs lacustres construisent des boites à pédiluve amovibles à fixer à l'entrée des logements dans les élevages en claustration partielle et totale. La limitation des contacts entre l'élevage et le matériel roulant, souvent recommandée dans les mesures de biosécurité classiques, est irréalisable en milieu lacustre, car pour avoir accès à l'élevage pour la distribution d'aliment, la vente des animaux, le nettoyage de la loge, etc., l'éleveur doit accéder jusqu'à l'entrée de l'élevage en claustration (partielle et totale) avec sa pirogue. Il faudrait alors désinfecter régulièrement ce matériel multi-usages (Penrith et al., 2011). Les éleveurs utilisant la claustration 
partielle devraient temporairement supprimer la liberté saisonnière en cas de foyers de PPA déclarés dans le voisinage. Ceux utilisant la conduite en liberté devraient passer, en cas de foyers de PPA, à la claustration partielle améliorée (sans liberté des porcs).

Enfin, l'apport des autorités locales pourrait être de mettre en place des fosses à cadavres pour centraliser les animaux morts afin d'empêcher les éleveurs de manipuler les porcs morts de la PPA et de jeter leurs cadavres n'importe où. Ils pourraient également assurer la fourniture de chaux vive pour chaque dépôt dans la fosse. En agissant ainsi, les autorités contribueraient aussi à la résolution des problèmes de santé publique associés à la dégradation des cadavres jetés dans l'environnement. L'utilisation de fosses pour la destruction des cadavres des animaux morts de la PPA a été déjà suggérée par Bora et al. (2020).

Les difficultés souvent observées au niveau des mesures de biosécurité sont le manque d'ajustement de ces mesures à la situation réelle, aussi bien dans leur conception que dans leur mise en œuvre effective. C'est pourquoi les mesures proposées ici après analyse de nos résultats devront être discutées avec les éleveurs et les autorités locales avant de pouvoir constituer la base de futures mesures de biosécurité et afin de réunir toutes les conditions de leur application.

\section{Conclusion}

L'étude a permis de constater que trois types d'élevages sont pratiqués dans les milieux lacustres. Ce sont l'élevage en claustration totale, l'élevage en claustration partielle et l'élevage en liberté. L'absence de mesures de biosécurité dans ces élevages engendre des problèmes sanitaires et surtout contribue à maintenir la PPA. De même, les transactions (vente et achat d'animaux) et la circulation de l'eau du fleuve peuvent faciliter la diffusion de la PPA entre les élevages lacustres et les élevages situés sur la terre ferme. Tous les types d'élevages sont exposés à cette maladie. Les pratiques d'élevage en milieu lacustre comportent beaucoup de facteurs de risques de diffusion du virus de la PPA dans ce milieu et dans les élevages sur la terre ferme. La menace de la PPA, dont tous les éleveurs ont pu mesurer l'impact, est aujourd'hui un levier pour faire avancer les débats sur l'amélioration des pratiques d'élevage, comme la nécessité du passage de tous les élevages en claustration totale lors de la présence de foyers de PPA. Les améliorations à apporter aux pratiques d'élevage en milieu lacustre devraient logiquement impliquer la mise en place de mesures de biosécurité spécifiques à cet environnement. Les résultats de cette étude ont permis de faire des propositions de mesures que tous les éleveurs, quel que soit leur mode d'élevage, pourraient mettre en application et que les autorités locales pourraient soutenir efficacement. Le progrès à atteindre au niveau des élevages n'est pas le passage de tous les élevages en claustration totale permanente ; c'est de réfléchir à un élevage en claustration partielle amélioré, avec des logements et des modalités de conduite adaptés, en tenant compte des mesures de biosécurité proposées. Ces élevages en claustration partielle et en liberté, une fois améliorés, permettraient de sauvegarder l'activité d'élevage, et en particulier celle des femmes rurales, et de maintenir la diversité génétique en préservant les animaux de type génétique local, tout en limitant les risques liés à la PPA.
Remerciements. Nous remercions les éleveurs qui ont voulu répondre à nos questions et les relecteurs pour l'amélioration de la première version de cet article.

\section{Références}

Agbokounou A, Ahounou G, Youssao I, Mensah A, Koutinhouin B, Hornic JL. 2016. Caractéristiques de l'élevage du porc local d'Afrique. Journal of Animal \& Plant Sciences 30(1): 4701-4713.

Asambe A, Sackey AKB, Tekdek LB. 2019. Sanitary measures in piggeries, awareness, and risk factors of African swine fever in Benue State, Nigeria. Tropical Animal Health and Production 51 (4): 997-1001. https://doi.org/10.1007/s11250-018-1764-7.

Bora M, Bora DP, Manu M, Barman NN, Dutta LJ, Kumar PP, et al. 2020. Assessment of risk factors of African swine fever in India: perspectives on future outbreaks and control strategies. Pathogens 9(12): 1-18. https://doi.org/10.3390/pathogens9121044.

Brown AA, Penrith ML, Fasina FO, Beltran-Alcrudo D. 2018. The African swine fever epidemic in West Africa, 1996-2002. Transboundary Emerging Diseases 65: 64-76. https://doi.org/ 10.1111/tbed.12673.

Costard S, Porphyre V, Messad S, Rakotondrahanta S, Vidon H, Roger F, et al. 2009. Multivariate analysis of management and biosecurity practices in smallholder pig farms in Madagascar. Preventive Veterinary Medicine 92: 199-209. https://doi.org/ 10.1016/j.prevetmed.2009.08.010.

Couacy-Hymann E. 2019. African swine fever in sub-Saharan African countries. In: Kardjadj M, Diallo A, Lancelot R, eds. Transboundary animal diseases in Sahelian Africa and connected regions. Switzerland: Springer, Cham, pp. 323-344. https://doi.org/ 10.1007/978-3-030-25385-1 16.

DE. 2019. Rapport d'activité. Direction de l'élevage, 86 p.

Dixon LK, Stahl K, Jori F, Vial L, Pfeiffer DU. 2020. African swine fever epidemiology and control. Annual Review of Animal Biosciences 8: 221-246. https://doi.org/10.1146/annurev-animal$021419-083741$.

Djenontin I. 2006a. Monographie de la commune de Sô-Ava. Programme d'Appui au Démarrage des Communes, Bénin, 42 p.

Djenontin I. 2006b. Monographie communale des Aguégués. Programme d'Appui au Démarrages des Communes, Bénin, $44 \mathrm{p}$.

Dotché OI, Ahounou S, Salifou C, Biobou R, Kiki P, Govoeyi B, et al. 2018. Selection and culling criteria for breeding boars and sows in pig farms from Oueme and Plateau departments in Benin. Revue d'élevage et de médecine vétérinaire des pays tropicaux 71(1-2): 47-57. https://doi.org/10.19182/remvt.31224.

Dotché OI, Idohou S, Dahouda M, Kiki SP, Govoeyi B, AntoineMoussiaux $\mathrm{N}$, et al. 2019. Crossbreeding and consanguinity management in pig farms in the departments of Ouémé and Plateau in Benin. Veterinary World 12(11): 1816-1825. https://doi.org/ 10.14202/vetworld.2019.1816-1825.

Eblé PL, Hagenaars TJ, Weesendorp E, Quak S, Moonen-Leusen HW, Loeffen WLA. 2019. Transmission of African Swine Fever Virus via carrier (survivor) pigs does occur. Veterinary Microbiology 237: 108345. https://doi.org/10.1016/j.vetmic.2019.06.018.

Fasina FO, Kissinga H, Mlowe F, Mshang'a S, Matogo B, Mrema A, et al. 2020. Drivers, risk factors and dynamics of African swine fever outbreaks, southern highlands, Tanzania. Pathogens 9(3): 155. https://doi.org/10.3390/pathogens 9030155.

Gulenkin VM, Korennoy FI, Karaulov AK, Dudnikov SA. 2011. Cartographical analysis of African swine fever outbreaks in the territory of the Russian Federation and computer modeling of the basic reproduction ratio. Preventive Veterinary Medicine 102(3): 167-174. https://doi.org/10.1016/j.prevetmed.2011.07.004. 
Kiki PS, Dahouda M, Toléba SS, Ahounou GS, Dotché IO, Govoeyi B, et al. 2018. Pig feeding management and pig farming constraints in Southern Benin. Revue d'élevage et de médecine vétérinaire des pays tropicaux 71(1-2): 67-74. https://doi.org/10.19182/remvt.31223.

Kiki PS, Dahouda M, Agbokounou A, Bonou G, Dotché OI, Govoeyi B, et al. 2019. Effect of energy and protein contents variation in diets on the immune status and growth performance of growing and fattening pigs. Journal of Agricultural Science and Food Technology 5(9): 218-228. https://doi.org/10.36630/jasft 19055.

Kouthinhouin GB, Youssao AKI, Toleba SS, Kpodekon TM, Ahounou GS, Bonou AG, et al. 2009. Effet du mode d'élevage sur la prolificité des truies de race locale du Bénin et la viabilité de leurs porcelets, de la naissance au sevrage. International Journal of Biological and Chemical Sciences 3(4): 819-829. https://doi.org/ 10.4314/ijbcs.v3i4.47188.

Martínez-López B, Perez AM, Feliziani F, Rolesu S, Mur L, SanchezVizcaino JM. 2015. Evaluation of the risk factors contributing to the African swine fever occurrence in Sardinia, Italy. Frontiers in Microbiology 6: 1-10. https://doi.org/10.3389/fmicb.2015.00314.

Mazur-Panasiuk N, Zmudzki J, Woźniakowski G. 2019. African swine fever virus - persistence in different environmental conditions and the possibility of its indirect transmission. Journal of Veterinary Research 63(3): 303-310. https://doi.org/10.2478/ jvetres-2019-0058.

Ohouko F, Koudouvo K, Dougnon TJ, Agbonon A, Youssao I, Farougou S, et al. 2020. African swine fever in Benin and prevalence of the disease in Southern Benin: a retrospective study (2014-2018). Journal of Advanced Veterinary and Animal Research 7(1): 464-470. https://doi.org/10.5455/javar.2020.g442.

Ossebi W, Ayssiwede SB, Nimbona F, Malou R, Djetiin AE, Diop M, et al. 2018. Obstacles to the development of the pig value chain in Casamance (Senegal): what do actors of the subsector say? Revue d'élevage et de médecine vétérinaire des pays tropicaux 71(1-2): 15-22. https://doi.org/10.19182/remvt.31286.

Penrith ML, Guberti V, Depner K, Lubroth J, 2011. Préparation des plans d'intervention contre la peste porcine africaine. Rome (Italie): FAO, $84 \mathrm{p}$.

Penrith ML, Vosloo W, Jori F, Bastos ADS. 2013. African swine fever virus eradication in Africa. Virus Research 173(1): 228-246. https://doi.org/10.1016/j.virusres.2012.10.011.

Tohoun E. 2017. Caractéristiques de l'élevage porcin dans les Aguégués et perspectives d'amélioration. Université d'AbomeyCalavi, DPSA/EPAC, $56 \mathrm{p}$.

Citation de l'article : Dotché OI, Bio Seydou K, Ahouanse AGG, Tohoun E, Bankolé BC, Youssao Abdou Karim I. 2021. Comment concevoir la biosécurité des élevages porcins des milieux lacustres du sud Bénin pour réduire le risque de maintien et de dissémination de la peste porcine africaine? Cah. Agric. 30: 32. 